\title{
Numerical solution of nonlinear fuzzy Fredholm integral equations of the second kind using hybrid of block-pulse functions and Taylor series
}

\author{
Mahdy Baghmisheh and Reza Ezzati ${ }^{*}$
}

${ }^{*}$ Correspondence: ezati@kiau.ac.ir ${ }^{2}$ Department of Mathematics, Karaj Branch, Islamic Azad University, Karaj, Iran

Full list of author information is available at the end of the article

\begin{abstract}
The main purpose of this paper is to introduce a method of successive approximations in terms of a hybrid of Taylor series and a block-pulse function, which is given for solving nonlinear fuzzy Fredholm integral equations of the second kind and the error estimate of the approximation solution. Finally, two numerical examples are presented to show the accuracy of the proposed method.
\end{abstract}

Keywords: nonlinear fuzzy Fredholm integral equation; hybrid of block-pulse function and Taylor series

\section{Introduction}

The study of fuzzy integral equations (FIE) from both theoretical and numerical points of view has been developed in recent years after a distinct study of the existence of a unique solution for fuzzy Fredholm integral equations had been carried out in [1]. The Banach fixed point theorem is the main tool in studying the existence and uniqueness of the solution for fuzzy integral equations which can be found in [2-4]. Numerical procedures for solving fuzzy integral equations of the second kind, based on the method of successive approximations and other iterative techniques, have been investigated in [5-9]. The Adomian decomposition method is used in [10-12], as well as quadrature rules, fuzzy Bernstein polynomials, fuzzy wavelet, and Nystorm techniques were applied to fuzzy integral equations of the second kind in [13-18].

Recently, Bica and Popescu $[19,20]$ applied the method of successive approximations for the fuzzy Hammerstein integral equation. Ezzati and Ziari [21] proved the convergence of the method of successive approximations for solving nonlinear fuzzy Fredholm integral equations of the second kind, and they proposed an iterative procedure based on the trapezoidal quadrature. Mirzaee [22] obtained an approximate solution for the linear Fredholm fuzzy integral equations of the second kind by the hybrid of block-pulse function and Taylor series (HBT).

In this paper, we approximate the fuzzy function by the hybrid Taylor and block-pulse functions (HBT) and estimate the error approximation. Also, an iterative procedure is

\section{Springer}

(c) 2015 Baghmisheh and Ezzati; licensee Springer. This is an Open Access article distributed under the terms of the Creative Commons Attribution License (http://creativecommons.org/licenses/by/4.0), which permits unrestricted use, distribution, and reproduction in any medium, provided the original work is properly credited. 
constructed based on HBT for solving nonlinear Fredholm fuzzy integral equations,

$$
F(t)=f(t) \oplus(F R) \int_{a}^{b} k(s, t) \odot G(F(s)) d s, \quad t \in[a, b] .
$$

We prove the convergence of this successive approximation method, and for demonstrating the accuracy of the proposed method we solve some numerical examples.

\section{Preliminaries}

Firstly, we present the basic concepts for fuzzy numbers and fuzzy-number-valued functions.

Definition 2.1 [23] A fuzzy number is a function $u: \mathbb{R} \rightarrow[0,1]$ satisfying the following properties:

(i) $u$ is normal, i.e. $\exists x_{0} \in \mathbb{R}$ with $u\left(x_{0}\right)=1$.

(ii) $u$ is a convex fuzzy set, i.e. $u(\lambda x+(1-\lambda) y) \geq \min \{u(x), u(y)\}, \forall x, y \in \mathbb{R}, \lambda \in[0,1]$.

(iii) $u$ is upper semi-continuous on $\mathbb{R}$.

(iv) $[u]_{0}=\overline{\{x \in \mathbb{R}: u(x)>0\}}$ is a compact interval, where $\bar{A}$ is the closure of the set $A$.

The set of all fuzzy real numbers is denoted by $\mathbb{R}_{\mathcal{F}}$. Any real number $a \in \mathbb{R}$ can be interpreted as a fuzzy number $\tilde{a}=\chi_{\{a\}}$ and therefore $\mathbb{R} \subset \mathbb{R}_{\mathcal{F}}$.

For $0<r \leq 1$, we denote $[u]_{r}=\{x \in \mathbb{R}: u(x) \geq r\}$ the $r$-level (or simply the $r$-cut) set of $u$ which is a closed interval (see [24]) and $[u]_{r}=\left[\underline{u}_{r}, \bar{u}_{r}\right], \forall r \in[0,1]$. This leads to the usual parametric representation of a fuzzy number.

Proposition 2.1 [25] A fuzzy number $u$ is completely determined by any ordered pair $u=$ $(\underline{u}(r), \bar{u}(r))$ of functions $\underline{u}, \bar{u}:[0,1] \rightarrow \mathbb{R}$ defining the three conditions:

(i) $\underline{u}: r \rightarrow \underline{u}_{r} \in \mathbb{R}$ is a bounded monotonic non-decreasing left-continuous function $\forall r \in] 0,1]$ and right-continuous for $r=0$;

(ii) $\bar{u}: r \rightarrow \bar{u}_{r} \in \mathbb{R}$ is a bounded monotonic non-increasing left-continuous function $\forall r \in] 0,1]$ and right-continuous for $r=0$;

(iii) $\underline{u}_{1} \leq \bar{u}_{1}$ for $r=1$, which implies $\underline{u}_{r} \leq \bar{u}_{r}, \forall r \in[0,1]$.

For $u, v \in \mathbb{R}_{\mathcal{F}}, k \in \mathbb{R}$, the addition and the scalar multiplication are defined as follows:

(1) $[u \oplus v]_{r}=[u]_{r}+[v]_{r}=\left[\underline{u}_{r}+\underline{v}_{r}, \bar{u}_{r}+\bar{v}_{r}\right], \forall r \in[0,1]$,

(2) $[k \odot u]_{r}=k \cdot[u]_{r}= \begin{cases}{\left[k \underline{u}_{r}, k \bar{u}_{r}\right],} & \text { if } k \geq 0, \\ \left.k \bar{u}_{r}, k \underline{u}_{r}\right], & \text { if } k<0 \text {. }\end{cases}$

The subtraction of fuzzy numbers $u \ominus v$ is defined as the addition $u \oplus(-v)$ where $(-v)=$ $(-1) \odot v$.

The standard Hukuhara difference (H-difference $\ominus_{H}$ ) is defined by $u \ominus_{H} v=w \Longleftrightarrow u=$ $v \oplus w$; if $u \ominus_{H} v$ exists, its $r$-cuts are $\left[u \ominus_{H} v\right]_{r}=\left[\underline{u}_{r}-\underline{v}_{r}, \bar{u}_{r}-\bar{v}_{r}\right]$. It is well known that $u \ominus_{H} u=\tilde{0}$ for all fuzzy numbers $u$, but $u \ominus u \neq \tilde{0}$.

Definition 2.2 [26] Let $u=(\underline{u}(r), \bar{u}(r)), v=(\underline{v}(r), \bar{v}(r)) \in \mathbb{R}_{\mathcal{F}}$ be fuzzy numbers with positive support $($ i.e. $\underline{u}(0)>0, \underline{v}(0)>0)$. The product $c=u \otimes v=(\underline{(u \otimes v)}(r), \overline{(u \otimes v)}(r)) \in$ $C[0,1] \times C[0,1]$ is defined by $\underline{(u \otimes v)}(r)=\underline{u}(r) \cdot \underline{v}(r)$ and $\overline{(u \otimes v)}(r)=\overline{\bar{u}(r)} \cdot \bar{v}(r), \forall r \in[0,1]$.

Lemma $2.1[5,24]$ The following algebraic properties hold: 
(i) $u \oplus(v \oplus w)=(u \oplus v) \oplus w$ and $u \oplus v=v \oplus u$ for any $u, v, w \in \mathbb{R}_{\mathcal{F}}$,

(ii) $u \oplus \tilde{0}=\tilde{0} \oplus u=u$ for any $u \in \mathbb{R}_{\mathcal{F}}$,

(iii) with respect to $\tilde{0}$, none of $u \in \mathbb{R}_{\mathcal{F}} \backslash \mathbb{R}, u \neq \tilde{0}$ has an opposite in $\left(\mathbb{R}_{\mathcal{F}}, \oplus\right)$,

(iv) for any $a, b \in \mathbb{R}$ with $a, b \geq 0$ or $a, b \leq 0$, and any $u \in \mathbb{R}_{\mathcal{F}}$ we have $(a+b) \odot u=a \odot u \oplus b \odot u$,

(v) for any $a \in \mathbb{R}$ and any $u, v \in \mathbb{R}_{\mathcal{F}}$ we have $a \odot(u \oplus v)=a \odot u \oplus a \odot v$,

(vi) for any $a, b \in \mathbb{R}$ and any $u \in \mathbb{R}_{\mathcal{F}}$ we have $a \odot(b \odot u)=(a b) \odot u$ and $1 \odot u=u$.

As a distance between fuzzy numbers, we use the Hausdorff metric (see [13]) defined by

$$
D(u, v)=\sup _{r \in[0,1]}\left\{\max \left(\left|\underline{u}_{r}-\underline{v}_{r}\right|,\left|\bar{u}_{r}-\bar{v}_{r}\right|\right)\right\}
$$

for any $u, v \in \mathbb{R}_{\mathcal{F}}$.

Lemma 2.2 [24] The Hausdorff metric has the following properties:

(i) $\left(\mathbb{R}_{\mathcal{F}}, D\right)$ is a complete metric space,

(ii) $D(u \oplus w, v \oplus w)=D(u, v), \forall u, v, w \in \mathbb{R}_{\mathcal{F}}$,

(iii) $D(u \oplus v, w \oplus e) \leq D(u, w)+D(v, e), \forall u, v, w, e \in \mathbb{R}_{\mathcal{F}}$,

(iv) $D(u \oplus v, \tilde{0}) \leq D(u, \tilde{0})+D(v, \tilde{0}), \forall u, v \in \mathbb{R}_{\mathcal{F}}$,

(v) $D(k \odot u, k \odot v)=|k| D(u, v), \forall u, v \in \mathbb{R}_{\mathcal{F}}, \forall k \in \mathbb{R}$.

Lemma 2.3 [27] For any $k_{1}, k_{2} \in \mathbb{R}$ with $k_{1} \cdot k_{2} \geq 0$ and any $u \in \mathbb{R}_{\mathcal{F}}$ we have

$$
D\left(k_{1} \odot u, k_{2} \odot u\right)=\left|k_{1}-k_{2}\right| D(u, \tilde{0}) .
$$

Remark 2.1 The properties (iv) in Lemma 2.2 suggest the definition of a function $\|\cdot\|$ : $\mathbb{R}_{\mathcal{F}} \rightarrow \mathbb{R}$ by $\|u\|=D(u, \tilde{0})$, which has the properties of the usual norms. In [5] the properties of this function are presented as follows:

(i) $\|u\| \geq 0, \forall u \in \mathbb{R}_{\mathcal{F}}$, and $\|u\|=0$ iff $u=\tilde{0}$,

(ii) $\|\lambda \odot u\|=|\lambda| \cdot\|u\|$ and $\|u \oplus v\| \leq\|u\|+\|v\|, \forall u, v \in \mathbb{R}_{\mathcal{F}}, \forall \lambda \in \mathbb{R}$,

(iii) $|\|u\|-\|v\|| \leq D(u, v)$ and $D(u, v) \leq\|u\|+\|v\|, \forall u, v \in \mathbb{R}_{\mathcal{F}}$.

We see that $\left(\mathbb{R}_{\mathcal{F}}, \oplus, \odot,\|\cdot\|\right)$ is not a normed space because $\left(\mathbb{R}_{\mathcal{F}}, \oplus\right)$ is not a group.

Definition 2.3 [25] Given two fuzzy numbers $u, v \in \mathbb{R}_{\mathcal{F}}$, the generalized Hukuhara difference (gH-difference for short) is the fuzzy number $w$, if it exists, such that

$$
u \ominus_{g H} v=w \Longleftrightarrow \begin{cases}\text { (i) } & u=v \oplus w, \text { or } \\ \text { (ii) } & v=u \ominus w .\end{cases}
$$

It is easy to show that (i) and (ii) are both valid if and only if $w$ is a crisp number. 
In terms of $r$-cuts we have $\left[u \ominus_{g H} v\right]_{r}=\left[\min \left\{\underline{u}_{r}-\underline{v}_{r}, \bar{u}_{r}-\bar{v}_{r}\right\}, \max \left\{\underline{u}_{r}-\underline{v}_{r}, \bar{u}_{r}-\bar{v}_{r}\right\}\right]$, and if the H-difference exists, then $u \ominus_{H} v=u \ominus_{g H} v$; the conditions for the $w=u \ominus_{g H} v \in \mathbb{R}_{\mathcal{F}}$ are

$$
\begin{array}{cl}
\text { case (i) } & \left\{\begin{array}{l}
\underline{w}_{r}=\underline{u}_{r}-\underline{v}_{r} \text { and } \bar{w}_{r}=\bar{u}_{r}-\bar{v}_{r} \\
\text { with } \underline{w}_{r} \text { increasing, } \bar{w}_{r} \text { decreasing, } \underline{w}_{r} \leq \bar{w}_{r}, \quad \forall r \in[0,1],
\end{array}\right. \\
\text { case (ii) } \quad\left\{\begin{array}{l}
\underline{w}_{r}=\bar{u}_{r}-\bar{v}_{r} \text { and } \bar{w}_{r}=\underline{u}_{r}-\underline{v}_{r} \\
\text { with } \underline{w}_{r} \text { increasing, } \bar{w}_{r} \text { decreasing, } \underline{w}_{r} \leq \bar{w}_{r}, \quad \forall r \in[0,1] .
\end{array}\right.
\end{array}
$$

Proposition 2.2 [28] Let $u, v \in \mathbb{R}_{\mathcal{F}}$ be two fuzzy numbers; then

(a) if the gH-difference exists, it is unique,

(b) $u \ominus_{g H} v=u \ominus_{H} v$ or $u \ominus_{g H} v=-\left(v \ominus_{H} u\right)$ whenever the expressions on the right exist; in particular, $u \ominus_{g H} u=u \ominus_{H} u=\tilde{0}$,

(c) if $u \ominus_{g H} v$ exists in the sense of (i), then $v \ominus_{g H} u$ exists in the sense of (ii) and vice versa,

(d) $(u \oplus v) \ominus_{g H} v=u$,

(e) $\tilde{0} \ominus_{g H}\left(u \ominus_{g H} v\right)=v \ominus_{g H} u$,

(f) $u \ominus_{g H} v=v \ominus_{g H} u=w$ if and only if $w=-w$; furthermore, $w=0$ if and only if $u=v$.

Definition 2.4 For any fuzzy-number-valued function $f: I \subset \mathbb{R} \rightarrow \mathbb{R}_{\mathcal{F}}$ we can define the functions $f_{-r}, \bar{f}_{r}: I \subset \mathbb{R} \longrightarrow \mathbb{R}, r \in[0,1]$ by $f_{-r}(t)=\underline{(f(t))_{r}}, \bar{f}_{r}(t)=\overline{(f(t))_{r}}, \forall t \in[0,1]$. These functions are the left and right $r$-level functions of $f$.

Definition 2.5 [29] A fuzzy-number-valued function $f:[a, b] \rightarrow \mathbb{R}_{\mathcal{F}}$ is said to be continuous at $t_{0} \in[a, b]$ if for each $\varepsilon>0$ there is $\delta>0$ such that $D\left(f(t), f\left(t_{0}\right)\right)<\varepsilon$ whenever $\left|t-t_{0}\right|<\delta$. If $f$ is continuous for each $t \in[a, b]$ then we say that $f$ is fuzzy continuous on $[a, b]$. A fuzzy number $u \in \mathbb{R}_{\mathcal{F}}$ is upper bound for a fuzzy-number-valued function

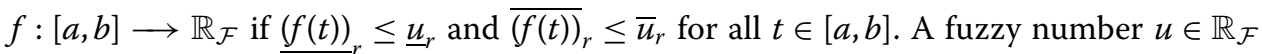
is a lower bound for a fuzzy-number-valued function $f:[a, b] \longrightarrow \mathbb{R}_{\mathcal{F}}$ if $\underline{u}_{r} \leq(f(t))$, and $\bar{u}_{r} \leq \overline{(f(t))_{r}}$ for all $t \in[a, b]$. A fuzzy-number-valued function $f:[a, b] \longrightarrow \mathbb{R}_{\mathcal{F}}$ is said to be bounded if it has a lower and an upper bound.

Remark 2.2 The above definition of the boundedness of a fuzzy-number-valued function can be expressed in the following equivalent form: $f:[a, b] \longrightarrow \mathbb{R}_{\mathcal{F}}$ is bounded iff there is $M \geq 0$ such that $D(f(t), \tilde{0}) \leq M$ for all $t \in[a, b]$. The constant $M$ can be chosen as $M \geq$ $\max \left\{\left|\underline{u}_{0}\right|,\left|\bar{u}_{0}\right|\right\}$.

Lemma 2.4 [29] Iff $:[a, b] \longrightarrow \mathbb{R}_{\mathcal{F}}$ is continuous then it is bounded and its supremum $\sup _{t \in[a, b]} f(t)$ must exist and is determined by $u \in \mathbb{R}_{\mathcal{F}}$ with $\underline{u}_{r}=\sup _{t \in[a, b]} f_{-r}(t)$ and $\bar{u}_{r}=$ $\sup _{t \in[a, b]} \bar{f}_{r}(t)$. A similar conclusion for the infimum is also true.

Let $C_{\mathcal{F}}[a, b]$, be the space of fuzzy continuous functions with the metric

$$
D^{*}(f, g)=\sup _{a \leq t \leq b} D(f(t), g(t)), \quad \forall f, g \in C_{\mathcal{F}}[a, b],
$$

which is called the uniform distance between fuzzy-number-valued functions. We see that $\left(C_{\mathcal{F}}[a, b], D^{*}\right)$ is a complete metric space and using Lemma 2.2 and Lemma 2.3 we can derive the corresponding properties of the metric $D^{*}$. 
Definition 2.6 [30] A fuzzy-number-valued function $f:[a, b] \longrightarrow \mathbb{R}_{\mathcal{F}}$ is said to be uniformly continuous on $[a, b]$, if for each $\varepsilon>0$ there is $\delta>0$ such that $D\left(f(t), f\left(t^{\prime}\right)\right)<\varepsilon$ whenever $t, t^{\prime} \in[a, b]$ with $\left|t-t^{\prime}\right|<\delta$.

Definition 2.7 [30] A fuzzy-number-valued function $f:[a, b] \longrightarrow \mathbb{R}_{\mathcal{F}}$ is said to level-

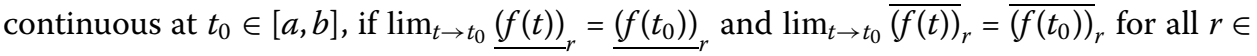
$[0,1]$. If $f$ is level-continuous at each $t \in[a, b]$, then we say that $f$ is level-continuous on $[a, b]$.

It is obvious that the continuity of a fuzzy-number-valued function implies the levelcontinuity, but the converse does not hold. However, the boundedness property holds for both types of continuity.

Definition 2.8 [5] Let $f:[a, b] \longrightarrow \mathbb{R}_{\mathcal{F}}$ be a bounded mapping. Then the function $\omega_{[a, b]}(f, \cdot): \mathbb{R}_{+} \cup 0 \rightarrow \mathbb{R}_{+}$

$$
\omega_{[a, b]}(f, \delta)=\sup \{D(f(x), f(y)): x, y \in[a, b],|x-y| \leq \delta\}
$$

is said to be the modulus of oscillation of $f$ on $[a, b]$.

If $f \in C_{\mathcal{F}}[a, b]$, then $\omega_{[a, b]}(f, \delta)$ is called uniform modulus of continuity of $f$.

Some properties of the modulus of oscillation are given below.

Proposition 2.3 [5] The following statements are true:

(i) $D(f(x), f(y)) \leq \omega_{[a, b]}(f,|x-y|)$ for any $x, y \in[a, b]$,

(ii) $\omega_{[a, b]}(f, \delta)$ is a non-decreasing mapping in $\delta$,

(iii) $\omega_{[a, b]}(f, 0)=0$,

(iv) $\omega_{[a, b]}\left(f, \delta_{1}+\delta_{2}\right) \leq \omega_{[a, b]}\left(f, \delta_{1}\right)+\omega_{[a, b]}\left(f, \delta_{2}\right)$ for any $\delta_{1}, \delta_{2} \geq 0$,

(v) $\omega_{[a, b]}(f, n \delta) \leq n \omega_{[a, b]}(f, \delta)$ for any $\delta \geq 0$ and $n \in \mathbb{N}$,

(vi) $\omega_{[a, b]}(f, \lambda \delta) \leq(\lambda+1) \omega_{[a, b]}(f, \delta)$ for any $\delta, \lambda \geq 0$,

(vii) If $[c, d] \subseteq[a, b]$ then $\omega_{[c, d]}(f, \delta) \leq \omega_{[a, b]}(f, \delta)$.

Based on the gH-difference, we obtain the following definition.

Definition 2.9 [25] Let $\left.x_{0} \in\right] a, b\left[\right.$ and $h$ be such that $\left.x_{0}+h \in\right] a, b[$, then the gH-derivative of a function $f:] a, b\left[\rightarrow \mathbb{R}_{\mathcal{F}}\right.$ at $x_{0}$ is defined as

$$
f_{g H}^{\prime}\left(x_{0}\right)=\lim _{h \rightarrow 0} \frac{1}{h}\left[f\left(x_{0}+h\right) \ominus_{g H} f\left(x_{0}\right)\right]
$$

If $f_{g H}^{\prime}\left(x_{0}\right) \in \mathbb{R}_{\mathcal{F}}$ satisfying Eq. (2.2) exists, we say that $f$ is generalized Hukuhara differentiable (gH-differentiable for short) at $x_{0}$.

Definition 2.10 [25] Let $\left.x_{0} \in\right] a, b\left[\right.$ and $h$ be such that $\left.x_{0}+h \in\right] a, b[$, then the level-wise gH-derivative (LgH-derivative for short) of a function $f:] a, b\left[\rightarrow \mathbb{R}_{\mathcal{F}}\right.$ at $x_{0}$ is defined as the set of interval-valued gH-derivatives, if they exist,

$$
f_{L g H}^{\prime}\left(x_{0}\right)_{r}=\lim _{h \rightarrow 0} \frac{1}{h}\left(\left[f\left(x_{0}+h\right)\right]_{r} \ominus_{g H}\left[f\left(x_{0}\right)\right]_{r}\right) .
$$


If $f_{L g H}^{\prime}\left(x_{0}\right)_{r}$ is a compact interval for all $r \in[0,1]$, we say that $f$ is level-wise generalized Hukuhara differentiable (LgH-differentiable for short) at $x_{0}$ and the family of intervals $\left\{f_{L g H}^{\prime}\left(x_{0}\right)_{r}: r \in[0,1]\right\}$ is the LgH-derivative of $f$ at $x_{0}$, denoted by $f_{L g H}^{\prime}\left(x_{0}\right)$.

Consequently, LgH-differentiability, as is level-wise continuity, is a necessary condition for gH-differentiability; but from Eq. (2.1), it is not sufficient.

The next result gives the analogous expression of the fuzzy gH-derivative in terms of the derivatives of the endpoints of the level sets. This result extends the result given in [31, Theorem 5] and it is a characterization of the gH-differentiability for an important class of fuzzy functions.

Proposition 2.4 [25] Let $f:] a, b\left[\rightarrow \mathbb{R}_{\mathcal{F}}\right.$ be such that $[f(x)]_{r}=\left[f_{-r}(x), \bar{f}_{r}(x)\right]$. Suppose that the functions $f_{-r}(x)$ and $\bar{f}_{r}(x)$ are real-valued functions, differentiable w.r.t. $x$, uniformly w.r.t. $r \in[0,1]$. Then the function $f(x)$ is gH-differentiable at a fixed $x \in] a, b[$ if and only if one of the following two cases holds:

(a) $\left(f_{-r}\right)^{\prime}(x)$ is increasing, $\left(\bar{f}_{r}\right)^{\prime}(x)$ is decreasing as a function of $r$, and $\left(f_{-1}\right)^{\prime}(x) \leq\left(\bar{f}_{1}\right)^{\prime}(x)$, or

(b) $\left(f_{-r}\right)^{\prime}(x)$ is decreasing, $\left(\bar{f}_{r}\right)^{\prime}(x)$ is increasing as a function of $r$, and $\left(\bar{f}_{1}\right)^{\prime}(x) \leq\left(f_{-1}\right)^{\prime}(x)$.

Also, $\forall r \in[0,1]$ we have

$$
\left[f_{g H}^{\prime}(x)\right]_{r}=\left[\min \left\{\left(f_{-r}\right)^{\prime}(x),\left(\bar{f}_{r}\right)^{\prime}(x)\right\}, \max \left\{\left(f_{-r}\right)^{\prime}(x),\left(\bar{f}_{r}\right)^{\prime}(x)\right\}\right]
$$

Definition 2.11 [25] Let $f:[a, b] \rightarrow \mathbb{R}_{\mathcal{F}}$ and $\left.x_{0} \in\right] a, b\left[\right.$ with $f_{-r}(x)$ and $\bar{f}_{r}(x)$ both differentiable at $x_{0}$.

We say that

- $f$ is (i)-gH-differentiable at $x_{0}$ if

$$
\text { (i) }\left[f_{g H}^{\prime}\left(x_{0}\right)\right]_{r}=\left[\left(f_{-r}\right)^{\prime}\left(x_{0}\right),\left(\bar{f}_{r}\right)^{\prime}\left(x_{0}\right)\right], \quad \forall r \in[0,1]
$$

- $f$ is (ii)-gH-differentiable at $x_{0}$ if

$$
\text { (ii) }\left[f_{g H}^{\prime}\left(x_{0}\right)\right]_{r}=\left[\left(\bar{f}_{r}\right)^{\prime}\left(x_{0}\right),\left(f_{-r}\right)^{\prime}\left(x_{0}\right)\right], \quad \forall r \in[0,1] \text {. }
$$

It is possible that $f:[a, b] \rightarrow \mathbb{R}_{\mathcal{F}}$ is gH-differentiable at $x_{0}$ and not (i)-gH-differentiable nor (ii)-gH-differentiable, as illustrated by Example 27 in [2].

Here $C_{\mathcal{F}}^{n}[a, b], n \geq 1$, denotes the space of $n$-times fuzzy continuously gH-differentiable functions from $[a, b]$ into $\mathbb{R}_{\mathcal{F}}$. (That is, there exist $f^{(k+1)}(x) \in \mathbb{R}_{\mathcal{F}}$ such that the limits in $D$-distance exist and

$$
f^{(k+1)}(x)=\lim _{h \rightarrow 0} \frac{1}{h}\left[f^{(k)}(x+h) \ominus_{g H} f^{(k)}(x)\right]
$$

for all $k=0,1, \ldots, n-1$.)

Denote the set of all functions $f \in C_{\mathcal{F}}^{n}[a, b], n \geq 1$, such that $f^{(k)}[a, b] \rightarrow \mathbb{R}_{\mathcal{F}}(k=$ $0,1, \ldots, n-1)$ is (i)-gH-differentiable on the interval $[a, b]$ by $C_{\mathcal{F}}^{n, 1}[a, b]$.

By Theorem 5.2 of $[21]$, for $f \in C_{\mathcal{F}}^{n, 1}[a, b]$ we obtain

$$
\left[f_{g H}^{(k)}(x)\right]_{r}=\left[\left(f_{-r}\right)^{(k)}(x),\left(\bar{f}_{r}\right)^{(k)}(x)\right], \quad k=0,1, \ldots, n \forall r \in[0,1], \forall x \in(a, b) .
$$


Proposition 2.5 [25] Iff $:[a, b] \rightarrow \mathbb{R}_{\mathcal{F}}$ is gH-differentiable (or right or left gH-differentiable) at $x_{0} \in[a, b]$ then it is level-wise continuous (or right or left level-wise continuous) at $x_{0}$.

Proposition 2.6 [25] The (i)-gH-derivative and (ii)-gH-derivative are additive operators, i.e., iff and $g$ are both (i)-gH-differentiable or both (ii)-gH-differentiable then

(i) $(f \oplus g)^{\prime}{ }_{(i)-g H}=f_{(i)-g H}^{\prime} \oplus g_{(i)-g H}^{\prime}$,

(ii) $(f \oplus g)^{\prime}{ }_{(i i)-g H}=f_{(i i)-g H}^{\prime} \oplus g^{\prime}{ }_{(i i)-g H}$.

In [24] the notion of a Henstock integral for fuzzy-number-valued functions is defined as follows.

Definition 2.12 [5] Let $f:[a, b] \rightarrow \mathbb{R}_{\mathcal{F}}$. For $\triangle_{n}: a=x_{0}<x_{1}<\cdots<x_{n-1}<x_{n}=b$ a partition of the interval $[a, b]$, we consider the points $\xi_{i} \in\left[x_{i-1}, x_{i}\right], i=1, \ldots, n$, and the function $\delta:[a, b] \rightarrow \mathbb{R}_{+}$. The partition $P=\left\{\left(\left[x_{i-1}, x_{i}\right] ; \xi_{i}\right) ; i=1, \ldots, n\right\}$ denoted by $P=\left(\triangle_{n}, \xi\right)$ is called $\delta$-fine iff $\left[x_{i-1}, x_{i}\right] \subseteq\left(\xi_{i}-\delta\left(\xi_{i}\right), \xi_{i}+\delta\left(\xi_{i}\right)\right)$. For $I \in \mathbb{R}_{\mathcal{F}}$, the function $f$ is fuzzy Henstock integrable on $[a, b]$ if for any $\varepsilon>0$ there is a function $\delta:[a, b] \rightarrow \mathbb{R}_{+}$such that for any partition $\delta$-fine $P, D\left(\sum_{i=1}^{n}\left(x_{i}-x_{i-1}\right) \odot f\left(\xi_{i}\right), I\right)<\varepsilon$. The fuzzy number $I$ is named the fuzzy Henstock integral of $f$ and will be denoted by $(F H) \int_{a}^{b} f(t) d t$.

When the function $\delta:[a, b] \rightarrow \mathbb{R}_{+}$is constant, then we obtain the Riemann integrability for fuzzy-number-valued functions (see [32]). In this case, $I \in \mathbb{R}_{\mathcal{F}}$ is called the fuzzy Riemann integral of $f$ on the interval $[a, b]$, denoted by $(F R) \int_{a}^{b} f(t) d t$. Consequently, the fuzzy Riemann integrability is a particular case of the fuzzy Henstock integrability, and therefore the properties of the integral $(F H)$ will be valid for the integral $(F R)$, too.

Lemma 2.5 [33] Let $f:[a, b] \rightarrow \mathbb{R}_{\mathcal{F}}$. Then $f$ is $(F H)$ integrable if and only if $f_{-r}$ and $\bar{f}_{r}$ are Henstock integrable for any $r \in[0,1]$. Furthermore, for any $r \in[0,1]$,

$$
\left[(F H) \int_{a}^{b} f(t) d t\right]_{r}=\left[(H) \int_{a}^{b} f_{r}(t) d t,(H) \int_{a}^{b} \bar{f}_{r}(t) d t\right] .
$$

Remark 2.3 If $f:[a, b] \rightarrow \mathbb{R}_{\mathcal{F}}$ is fuzzy continuous, then $f_{-r}$ and $\bar{f}_{r}$ are continuous for any $r \in[0,1]$ and consequently, they are Henstock integrable. According to Lemma 2.5 we infer that $f$ is $(F H)$ integrable.

Lemma 2.6 [5] Iff and $g$ are fuzzy Henstock integrable functions and if the function given by $D(f(t), g(t))$ is Lebesgue integrable, then

$$
D\left((F H) \int_{a}^{b} f(t) d t,(F H) \int_{a}^{b} g(t) d t\right) \leq(L) \int_{a}^{b} D(f(t), g(t)) d t .
$$

Theorem 2.1 [32] If $f, g:[a, b] \rightarrow \mathbb{R}_{\mathcal{F}}$ are $(F R)$ integrable fuzzy functions, and $\alpha, \beta$ are real numbers, then

$$
(F R) \int_{a}^{b}(\alpha \odot f(t) \oplus \beta \odot g(t)) d t=\alpha \odot(F R) \int_{a}^{b} f(t) d t \oplus \beta \odot(F R) \int_{a}^{b} g(t) d t .
$$


Remark 2.4 If $f:[a, b] \rightarrow \mathbb{R}_{\mathcal{F}}$ is fuzzy continuous, for a partition $\Delta: a=x_{0}<x_{1}<\cdots<$ $x_{n-1}<x_{n}=b$, according to [24], the fuzzy-Riemann integral has the property

$$
(F R) \int_{a}^{b} f(t) d t=\sum_{i=0}^{n-1} *(F R) \int_{t_{i}}^{t_{i+1}} f(t) d t
$$

where $\sum^{*}$ means addition with respect to $\oplus$ in $\mathbb{R}_{\mathcal{F}}$.

Definition 2.13 [5] For $L \geq 0$, a function $f:[a, b] \rightarrow \mathbb{R}_{\mathcal{F}}$ is $L$-Lipschitz if

$$
D(f(x), f(y)) \leq L|x-y|
$$

for any $x, y \in[a, b]$.

We present the following fuzzy Taylor theorem in one dimension.

Theorem 2.2 (see $[34, \mathrm{p} .51])$ Let $f \in C_{\mathcal{F}}^{n, 1}[a, b], n \geq 1,[\alpha, \beta] \subseteq[a, b] \subseteq \mathbb{R}$. Then

$$
\begin{gathered}
f(\beta)=f(\alpha) \oplus(\beta-\alpha) \odot f^{\prime}(\alpha) \oplus \cdots \oplus \frac{(\beta-\alpha)^{n-1}}{(n-1) !} \odot f^{(n-1)}(\alpha) \\
\oplus \frac{1}{(n-1) !} \odot(F R) \int_{\alpha}^{\beta}(\beta-t)^{n-1} \odot f^{(n)}(t) d t .
\end{gathered}
$$

The integral remainder is a fuzzy continuous function in $\beta$.

\section{Review of HBT}

Now, we recall some definitions, notations, and facts of the hybrid Taylor series and blockpulse functions and we generalize them to the fuzzy setting.

Definition 3.1 [35] Block-pulse functions $\varphi_{i}(t), i=1,2, \ldots, N$, on the interval $[0,1)$, are defined as

$$
\varphi_{i}(t)= \begin{cases}1, & \frac{i-1}{N} \leq t<\frac{i}{N} \\ 0, & \text { otherwise }\end{cases}
$$

where $N$ is an arbitrary positive integer.

The block-pulse functions on $[0,1)$ are disjoint, so for $i, j=1,2, \ldots, N$, we have $\varphi_{i}(t) \varphi_{j}(t)=$ $\delta_{i, j} \varphi_{i}(t)$, where $\delta_{i, j}$ is the Kronecker delta, also these functions have the property of orthogonality on [0,1). For more details see [36].

Consider the set of Taylor polynomials $T_{m}(t)=t^{m}, m=0,1,2, \ldots$. For $M$ being an arbitrary positive integer, hybrid Taylor block-pulse functions are defined as follows.

Definition 3.2 [36, 37] The set of hybrid Taylor block-pulse functions $h_{i j}(t), i=1, \ldots, N$; $j=0,1, \ldots, M$, on the interval $[0,1)$ is defined as

$$
h_{i j}(t)= \begin{cases}T_{j}(N t-(i-1)), & \frac{i-1}{N} \leq t<\frac{i}{N} \\ 0, & \text { otherwise }\end{cases}
$$

where $i$ and $j$ are the order of block-pulse functions and Taylor polynomials, respectively. 


\subsection{Function approximation}

For $f$ in $C_{\mathcal{F}}^{M, 1}[0,1]$, let us consider a fuzzy hybrid polynomial of degree $M-1$,

$$
T_{M}^{\mathcal{F}}(f)(t)=\sum_{i=1}^{N} * \sum_{j=0}^{M-1}{ }^{*} f_{i j} \odot h_{i j}(t)=F^{T} \odot H(t),
$$

where $h_{i j}(t)$ is defined in Eq. (3.1) and $f_{i j}$ are given by

$$
f_{i j}=\left.\frac{1}{N^{j} j !} f^{(j)}(t)\right|_{t=\frac{i-1}{N}} .
$$

Also, we have

$$
\begin{aligned}
& F=\left[f_{10}, \ldots, f_{1(M-1)}, f_{20}, \ldots, f_{2(M-1)}, \ldots, f_{N 0}, \ldots, f_{N(M-1)}\right]^{T} \\
& H(t)=\left[h_{10}(t), \ldots, h_{1(M-1)}(t), h_{20}(t), \ldots, h_{2(M-1)}(t), \ldots, h_{N 0}(t), \ldots, h_{N(M-1)}(t)\right]^{T} .
\end{aligned}
$$

Theorem 3.1 If $\in C_{\mathcal{F}}^{l, 1}[0,1]$ and there exists $K>0$, with $K=\sup _{0 \leq t \leq 1}\left\|f^{(l)}(t)\right\|_{\mathcal{F}}$, then

$$
D^{*}\left(T_{l}^{\mathcal{F}}(f), f\right) \leq \frac{K}{l ! N^{l}}
$$

Proof Let $t \in\left[0,1\left[\right.\right.$, so there exists $i \in 1,2, \ldots, N$ such that $t \in\left[\frac{i-1}{N}, \frac{i}{N}[\right.$. Then from Eqs. (3.1)-(3.3), we observe that

$$
\begin{aligned}
D\left(T_{l}^{\mathcal{F}}(f)(t), f(t)\right) & =D\left(\sum_{i=1}^{N} * \sum_{j=0}^{l-1} * f_{i j} \odot h_{i j}(t), f(t)\right) \\
& =D\left(\left.\sum_{j=0}^{l-1} * \frac{1}{N^{j} j !} f^{(j)}(t)\right|_{t=\frac{i-1}{N}} \odot T_{j}(N t-(i-1)), f(t)\right) \\
& =D\left(\sum_{j=0}^{l-1} * \frac{1}{j !}\left(t-\frac{i-1}{N}\right)^{j} \odot f^{(j)}\left(\frac{i-1}{N}\right), f(t)\right) \\
& =D\left(\frac{1}{(l-1) !} \odot(F R) \int_{\frac{i-1}{N}}^{t}(t-s)^{l-1} \odot f^{(l)}(s) d s, \tilde{0}\right) \\
& \leq \frac{1}{(l-1) !} \int_{\frac{i-1}{N}}^{t} D\left(f^{(l)}(s), \tilde{0}\right)(t-s)^{l-1} d s .
\end{aligned}
$$

Hence

$$
D\left(T_{l}^{\mathcal{F}}(f)(t), f(t)\right) \leq \frac{K}{l !}\left(t-\frac{i-1}{N}\right)^{l} \leq \frac{K}{l ! N^{l}}
$$

therefore

$$
D^{*}\left(T_{l}^{\mathcal{F}}(f), f\right) \leq \frac{K}{l ! N^{l}} .
$$

Remark 3.1 This shows that $\lim _{l, N \rightarrow \infty} D^{*}\left(T_{l}^{\mathcal{F}}(f), f\right)=0$. 


\section{Fuzzy integral equations}

We consider the nonlinear fuzzy Fredholm integral Eq. (1.1), where $k(s, t)$ is a positive crisp kernel function over the square $a \leq s, t \leq b, F(t)$ is a fuzzy-number-valued function and $G: R_{F} \rightarrow R_{F}$ is continuous. We assume that $k$ is continuous and therefore it is uniformly continuous with respect to $t$ and there exists $M>0$, such that $M=\max _{a \leq s, t \leq b}|k(s, t)|$.

Theorem 4.1 [21] Let the function $k(s, t)$ be continuous and positive for $a \leq s, t \leq b$, and function $f(t)$ be a fuzzy continuous in $[a, b]$.

Moreover, assume that there exists $L>0$, with

$$
D\left(G\left(F_{1}(u)\right), G\left(F_{2}(v)\right)\right) \leq L \cdot D\left(F_{1}(u), F_{2}(v)\right), \quad \forall u, v \in[a, b] .
$$

If $C=M L(b-a)<1$ then the fuzzy integral Eq. (1.1) has a unique solution $F^{*} \in X$, and it can be obtained by the following successive approximations method:

$$
\begin{aligned}
F_{0}(t) & =f(t), \\
F_{m}(t) & =f(t) \oplus(F R) \int_{a}^{b} k(s, t) \odot G\left(F_{m-1}(s)\right) d s, \quad \forall t \in[a, b], m \geq 1 .
\end{aligned}
$$

Moreover, the sequence of successive approximations, $\left(F_{m}\right)_{m \geq 1}$, converges to the solution $F^{*}$. Furthermore, the following error bound holds:

$$
D\left(F^{*}(t), F_{m}(t)\right) \leq \frac{C^{m+1}}{L(1-C)} M_{0}, \quad \forall t \in[a, b], m \geq 1,
$$

where $M_{0}=\sup _{a \leq t \leq b}\|G(f(t))\|_{F}$.

Now, we introduce the numerical method to find the approximate solution of the nonlinear fuzzy Fredholm integral equation as

$$
F(t)=f(t) \oplus(F R) \int_{0}^{1} k(s, t) \odot G(F(s)) d s, \quad t \in[0,1] .
$$

In this way, we consider the uniform partition of the interval $[0,1]$ :

$$
\triangle: 0=t_{0}<t_{1}<\cdots<t_{N-1}<t_{N}=1
$$

with $t_{i}=i h$, where $h=\frac{1}{N}$. Then the following iterative procedure gives the approximate solution of Eq. (4.3) in the point $t \in[0,1]$ :

$$
\begin{aligned}
y_{0}(t) & =f(t), \\
y_{m}(t) & =f(t) \oplus(F R) \int_{0}^{1} k(s, t) \odot T_{l}^{\mathcal{F}}\left(G\left(y_{m-1}\right)\right)(s) d s, \quad \forall t \in[0,1], m \geq 1 .
\end{aligned}
$$

The above recursive relation can be written in compact form as follows:

$$
\begin{aligned}
& y_{0}(t)=f(t), \\
& y_{m}(t)=f(t) \oplus \sum_{i=1}^{N} * \sum_{j=0}^{l-1}{ }^{*} H_{i j}(t) \odot g_{i j}^{(m-1)}, \quad \forall t \in[0,1], m \geq 1,
\end{aligned}
$$


where $g_{i j}^{(m-1)}$ is a fuzzy number defined by

$$
g_{i j}^{(m-1)}=\left.\frac{1}{N j j !}\left(\frac{d^{j} G\left(y_{m-1}(t)\right)}{d t^{j}}\right)\right|_{t=\frac{i-1}{N}}, \quad i=1,2, \ldots, N, j=0,1, \ldots, l-1,
$$

and

$$
H_{i j}(t)=\int_{0}^{1} k(s, t) h_{i j}(s) d s
$$

\section{Convergence analysis}

In this section, we analyze the convergence of the iterative procedure proposed for the solution of Eq. (4.3).

Theorem 5.1 Assume that Eq. (4.3) satisfies the following conditions:

(i) $f:[a, b] \rightarrow R_{F}$ is fuzzy continuous.

(ii) $k:[0,1] \times[0,1] \rightarrow R^{+}$is continuous and there exists $M>0$, such that $M=\max _{0 \leq s, t \leq 1}|k(s, t)|$.

(iii) $G: R_{F} \rightarrow R_{F}$ is fuzzy differentiable of order l, for positive integer number l. In addition, there exists $L>0$ such that

$$
D\left(G\left(F_{1}(u)\right), G\left(F_{2}(v)\right)\right) \leq L \cdot D\left(F_{1}(u), F_{2}(v)\right), \quad \forall u, v \in[0,1]
$$

where $L<M^{-1}$ and $F_{1}, F_{2}:[0,1] \rightarrow R_{\mathcal{F}}$.

Then the iterative procedure Eq. (4.5) converges to the unique solution of Eq. (4.3) F, and its error estimate is as follows:

$$
D^{*}\left(F, y_{m}\right) \leq \frac{M}{1-M L}\left(M_{0}(M L)^{m}+\frac{K}{N^{l} l !}\right)
$$

where $M_{0}=\sup _{0 \leq t \leq 1}\|G(f(t))\|_{\mathcal{F}}$,

$$
K=\max \left\{\sup _{0 \leq t \leq 1}\left\|\frac{d^{l} G\left(y_{n}(t)\right)}{d t^{l}}\right\|_{\mathcal{F}} ; 0 \leq n \leq m-1\right\} .
$$

Proof Since $F_{1}(t)=f(t) \oplus(F R) \int_{0}^{1} k(s, t) \odot G\left(F_{0}(s)\right) d s$, we have

$$
\begin{aligned}
D( & \left.F_{1}(t), y_{1}(t)\right) \\
= & D(f(t), f(t)) \\
& +D\left((F R) \int_{0}^{1} k(s, t) \odot G\left(F_{0}(s)\right) d s,(F R) \int_{0}^{1} k(s, t) \odot T_{l}^{\mathcal{F}}\left(G\left(y_{0}\right)\right)(s) d s\right) \\
\leq & \int_{0}^{1} D\left(k(s, t) \odot G(f(s)), k(s, t) \odot T_{l}^{\mathcal{F}}(G(f))(s)\right) d s \\
\leq & M \int_{0}^{1} D\left(G(f(s)), T_{l}^{\mathcal{F}}(G(f))(s)\right) d s .
\end{aligned}
$$


From Eq. (5.1) and Theorem 3.1, we obtain

$$
D\left(F_{1}(t), y_{1}(t)\right) \leq \frac{M K}{N^{l} l !}
$$

Now, since $F_{2}(t)=f(t) \oplus(F R) \int_{0}^{1} k(s, t) \odot G\left(F_{1}(s)\right) d s$, we conclude that

$$
\begin{aligned}
D & \left.F_{2}(t), y_{2}(t)\right) \\
& =D\left((F R) \int_{0}^{1} k(s, t) \odot G\left(F_{1}(s)\right) d s,(F R) \int_{0}^{1} k(s, t) \odot T_{l}^{\mathcal{F}}\left(G\left(y_{1}\right)\right)(s) d s\right) \\
& \leq M \int_{0}^{1} D\left(G\left(F_{1}(s)\right), T_{l}^{\mathcal{F}}\left(G\left(y_{1}\right)\right)(s)\right) d s \\
& \leq M \int_{0}^{1} D\left(G\left(F_{1}(s)\right), G\left(y_{1}(s)\right)\right) d s+M \int_{0}^{1} D\left(G\left(y_{1}(s)\right), T_{l}^{\mathcal{F}}\left(G\left(y_{1}\right)\right)(s)\right) d s .
\end{aligned}
$$

By using Eq. (5.2), Theorem 3.1, and the condition (iii), we obtain

$$
D\left(F_{2}(t), y_{2}(t)\right) \leq \frac{M^{2} L K}{N^{l} l !}+\frac{M K}{N^{l} l !}
$$

By induction, it is proven that

$$
D\left(F_{m}(t), y_{m}(t)\right) \leq\left(M^{m-1} L^{m-1}+M^{m-2} L^{m-2}+\cdots+M L+1\right) \frac{M K}{N^{l} l !}
$$

for each $t \in[0,1]$. Therefore

$$
D^{*}\left(F_{m}, y_{m}\right) \leq \frac{1-(M L)^{m}}{1-M L}\left(\frac{M K}{N^{l} l !}\right)
$$

Since $M L<1$, according to $\frac{1-(M L)^{m}}{1-M L} \leq \frac{1}{1-M L}$, for each $m \in N$, we get

$$
D^{*}\left(F_{m}, y_{m}\right) \leq \frac{1}{1-M L}\left(\frac{M K}{N^{l} l !}\right)
$$

Using Eq. (4.2), we obtain

$$
D\left(F(t), F_{m}(t)\right) \leq \frac{(M L)^{m+1}}{L(1-M L)} M_{0}, \quad \forall t \in[0,1], m \geq 1,
$$

where $M_{0}=\sup _{0 \leq t \leq 1}\|G(f(t))\|_{\mathcal{F}}$. So we have

$$
\begin{aligned}
D^{*}\left(F, y_{m}\right) & \leq D^{*}\left(F, F_{m}\right)+D^{*}\left(F_{m}, y_{m}\right) \\
& \leq \frac{(M L)^{m+1}}{L(1-M L)} M_{0}+\frac{1}{1-M L}\left(\frac{M K}{N^{l} l !}\right) \\
& \leq \frac{M}{1-M L}\left(M_{0}(M L)^{m}+\frac{K}{N^{l} l !}\right) .
\end{aligned}
$$


Remark 5.1 Since $M L<1$, it is easy to show that

$$
\lim _{\substack{m \rightarrow \infty \\ N \rightarrow \infty \\ l \rightarrow \infty}} D^{*}\left(F, y_{m}\right)=0
$$

That shows the convergence of the method.

\section{Numerical examples}

In this section, we apply the presented method in Section 4 for solving the fuzzy integral Eq. (4.3) in two examples. The approximate solution is calculated for different values of $N$, $l$ and $m$. Also, we compare the numerical solution obtained by using the proposed method with the exact solution. The computations associated with the examples were performed using Mathematica 7.

Example 6.1 [21] Consider the following nonlinear fuzzy Fredholm integral equation:

$$
F(t)=f(t) \oplus(F R) \int_{0}^{1} \frac{t s}{3} \odot(F(s))^{2} d s,
$$

where

$$
\begin{aligned}
k(s, t) & =\frac{t s}{3}, \quad s, t \in[0,1], \\
\underline{f}(t, r) & =\frac{11}{12} t-\frac{1}{6}(1-r)+\frac{1}{27} t(1-r)-\frac{1}{216} t(1-r)^{2}, \quad t, r \in[0,1], \\
\bar{f}(t, r) & =\frac{11}{12} t+\frac{1}{6}(1-r)-\frac{1}{27} t(1-r)-\frac{1}{216} t(1-r)^{2}, \quad t, r \in[0,1] .
\end{aligned}
$$

The exact solution is

$$
(\underline{F}(t, r), \bar{F}(t, r))=\left(t-\frac{1}{6}(1-r), t+\frac{1}{6}(1-r)\right) .
$$

\begin{tabular}{|c|c|c|c|c|}
\hline \multirow[t]{2}{*}{ r-level } & \multicolumn{2}{|c|}{$N=20, I=3, m=3$} & \multicolumn{2}{|c|}{$N=20, I=3, m=6$} \\
\hline & $\left|\underline{F}-\underline{y}_{m}\right|$ & $\left|\bar{F}-\bar{y}_{m}\right|$ & $\left|\underline{F}-\underline{y}_{m}\right|$ & $\left|\bar{F}-\bar{y}_{m}\right|$ \\
\hline 0.00 & $5.3401 \mathrm{E}-5$ & 4.95336E-4 & $1.16313 \mathrm{E}-7$ & 4.18483E-6 \\
\hline 0.25 & $7.4791 \mathrm{E}-5$ & $3.93359 \mathrm{E}-4$ & $2.00359 \mathrm{E}-7$ & $2.89065 \mathrm{E}-6$ \\
\hline 0.50 & $1.02649 \mathrm{E}-4$ & $3.08908 \mathrm{E}-4$ & $3.33720 \mathrm{E}-7$ & $1.96110 \mathrm{E}-6$ \\
\hline 0.75 & 1.38345E-4 & $2.39642 \mathrm{E}-4$ & $5.39466 \mathrm{E}-7$ & $0.30447 \mathrm{E}-6$ \\
\hline 1.00 & $1.83431 \mathrm{E}-4$ & $1.83431 \mathrm{E}-4$ & $8.49032 \mathrm{E}-7$ & 8.49032E-7 \\
\hline
\end{tabular}

The comparison of the HBT solution and the exact solution is shown in Table 1.

Example 6.2 Consider the following nonlinear fuzzy Fredholm integral equation:

$$
F(t)=f(t) \oplus(F R) \int_{0}^{1} s^{3} \exp (-t) \odot(F(s))^{2} d s
$$

Table 1 The accuracy on the level sets for Example 6.1 in $t=0.5$ 
Table 2 The accuracy on the level sets for Example 6.2 in $t=0.5$

\begin{tabular}{|c|c|c|c|c|}
\hline \multirow[t]{2}{*}{$\overline{r \text {-level }}$} & \multicolumn{2}{|c|}{$N=20, I=4, m=6$} & \multicolumn{2}{|c|}{$N=20, I=4, m=12$} \\
\hline & $\underline{\mid \boldsymbol{F}}-\underline{\boldsymbol{y}}_{\boldsymbol{m}} \mid$ & $\left|\bar{F}-\bar{y}_{m}\right|$ & $\left|\underline{F}-\underline{y}_{m}\right|$ & $\left|\bar{F}-\bar{y}_{m}\right|$ \\
\hline 0.00 & $2.72812 \mathrm{E}-4$ & $3.5242 \mathrm{E}-4$ & $5.40018 \mathrm{E}-7$ & $7.09831 E-7$ \\
\hline 0.25 & $2.81818 \mathrm{E}-4$ & $3.41478 \mathrm{E}-4$ & $5.57681 \mathrm{E}-7$ & $6.84684 \mathrm{E}-7$ \\
\hline 0.50 & $2.9108 \mathrm{E}-4$ & $3.30832 E-4$ & $5.76266 \mathrm{E}-7$ & $6.60765 E-7$ \\
\hline 0.75 & $3.00607 \mathrm{E}-4$ & $3.20476 \mathrm{E}-4$ & $5.95818 \mathrm{E}-7$ & $6.38016 \mathrm{E}$ \\
\hline 1.00 & $3.10403 E-4$ & $3.10403 \mathrm{E}-4$ & $6.16384 \mathrm{E}-7$ & $6.16384 E-7$ \\
\hline
\end{tabular}

where

$$
\begin{aligned}
k(s, t)= & s^{3} \exp (-t), \quad s, t \in[0,1], \\
f(t, r)= & \cosh t+\exp (-t)\left(\frac{475 \exp (-2)}{512}-\frac{\exp (2)}{32}-\frac{139}{512}+\frac{19 \exp (-2) r}{128}\right. \\
& \left.+\frac{9 r}{640}+\frac{19 \exp (-2) r^{2}}{3,200}-\frac{3 r^{2}}{3,200}\right), \quad t, r \in[0,1], \\
\bar{f}(t, r)= & \cosh t+\exp (-t)\left(\frac{15,979 \exp (-2)}{12,800}-\frac{\exp (2)}{32}-\frac{3,163}{12,800}-\frac{551 \exp (-2) r}{3,200}\right. \\
& \left.-\frac{33 r}{3,200}+\frac{19 \exp (-2) r^{2}}{3,200}-\frac{3 r^{2}}{3,200}\right), \quad t, r \in[0,1] .
\end{aligned}
$$

The exact solution in this case is given by

$$
(\underline{F}(t, r), \bar{F}(t, r))=\left(\cosh t+\frac{1}{40}(5+2 r) \exp (-t), \cosh t+\frac{1}{40}(9-2 r) \exp (-t)\right) .
$$

The comparison of the HBT solution and the exact solution is shown in Table 2.

\section{Conclusion}

In this paper, we have suggested an iterative procedure by utilizing fuzzy HBT to solve the nonlinear Ferdholm fuzzy integral Eq. (4.3). The error estimate of the approximated function was obtained by using the fuzzy Taylor theorem [34] for the function which is (i)-gH-differentiable. The error estimate of the present method is proved; for getting the best approximating solution of the equation, the number $N$ and the degree of the fuzzy hybrid polynomial $l$ must be chosen sufficiently large. The analyzed example illustrates the ability and reliability of the fuzzy HBT method for Eq. (4.3).

Competing interests

The authors declare that they have no competing interests.

Authors' contributions

All authors contributed equally to the writing of this paper. All authors read and approved the final manuscript.

Author details

'Department of Mathematics, Science and Research Branch, Islamic Azad University, Tehran, Iran. ${ }^{2}$ Department of Mathematics, Karaj Branch, Islamic Azad University, Karaj, Iran.

Received: 3 November 2014 Accepted: 27 January 2015 Published online: 24 February 2015

References

1. Mordeson, J, Newman, W: Fuzzy integral equations. Inf. Sci. 87, 215-229 (1995) 
2. Balachandran, K, Kanagarajan, K: Existence of solutions of general nonlinear fuzzy Volterra-Fredholm integral equations. J. Appl. Math. Stoch. Anal. 3, 333-343 (2005)

3. Park, JY, Han, HK: Existence and uniqueness theorem for a solution of fuzzy Volterra integral equations. Fuzzy Sets Syst. 105, 481-488 (1999)

4. Subrahmanyam, PV, Sudarsanam, SK: A note on fuzzy Volterra integral equations. Fuzzy Sets Syst. 81, 237-240 (1996)

5. Bede, B, Gal, SG: Quadrature rules for integrals of fuzzy-number-valued functions. Fuzzy Sets Syst. 145, 359-380 (2004)

6. Bica, AM: Error estimation in the approximation of the solution of nonlinear fuzzy Fredholm integral equations. Inf. Sci. 178, 1279-1292 (2008)

7. Friedman, M, Ma, M, Kandel, A: Numerical solutions of fuzzy differential and integral equations. Fuzzy Sets Syst. 106, 35-48 (1999)

8. Friedman, M, Ma, M, Kandel, A: Solutions to fuzzy integral equations with arbitrary kernels. Int. J. Approx. Reason. 20, 249-262 (1999)

9. Wu, C, Song, S, Wang, H: On the basic solutions to the generalized fuzzy integral equation. Fuzzy Sets Syst. 95 255-260 (1998)

10. Abbasbandy, S, Allahviranloo, T: The Adomian decomposition method applied to the fuzzy system of Fredholm integral equations of the second kind. Int. J. Uncertain. Fuzziness Knowl.-Based Syst. 14, 101-110 (2006)

11. Babolian, E, Sadeghi Goghary, H, Abbasbandy, S: Numerical solution of linear Fredholm fuzzy integral equations of the second kind by Adomian method. Appl. Math. Comput. 161, 733-744 (2005)

12. Rouhparvar, H, Allahviranloo, T, Abbasbandy, S: Existence and uniqueness of fuzzy solution for linear Volterra fuzzy integral equations proved by Adomian decomposition method. ROMAI J. 5(2), 153-161 (2009)

13. Abbasbandy, S, Babolian, E, Alavi, M: Numerical method for solving linear Fredholm fuzzy integral equations of the second kind. Chaos Solitons Fractals 31(1), 138-146 (2007)

14. Khezerloo, M, Allahviranloo, T, Salahshour, S, Khorasani Kiasari, M, Haji Ghasemi, S: Application of Gaussian quadratures in solving fuzzy Fredholm integral equations. In: Information Processing and Management of Uncertainty in Knowledge-Based Systems. Applications. Communications in Computer and Information Science, vol. 81, pp. 481-490 (2010)

15. Salehi, P, Nejatiyan, M: Numerical method for nonlinear fuzzy Volterra integral equations of the second kind. Int. J. Ind. Math. 3(3), 169-179 (2011)

16. Mokhtarnejad, F, Ezzati, R: The numerical solution of nonlinear Hammerstein fuzzy integral equations by using fuzzy wavelet like operator. J. Intell. Fuzzy Syst. doi:10.3233/IFS-141446

17. Ziari, S, Ezzati, R, Abbasbandy, S: Numerical solution of linear fuzzy Fredholm integral equations of the second kind using fuzzy Haar wavelet. In: Advances in Computational Intelligence. Communications in Computer and Information Science, vol. 299 (2012)

18. Ezzati, R, Ziari, S: Numerical solution and error estimation of fuzzy Fredholm integral equation using fuzzy Bernstein polynomials. Aust. J. Basic Appl. Sci. 5(9), 2072-2082 (2011)

19. Bica, AM, Popescu, C: Approximating the solution of nonlinear Hammerstein fuzzy integral equations. Fuzzy Sets Syst. 245, 1-17 (2014)

20. Bica, AM, Popescu, C: Numerical solutions of the nonlinear fuzzy Hammerstein-Volterra delay integral equations. Inf. Sci. 223, 236-255 (2013)

21. Ezzati, R, Ziari, S: Numerical solution of nonlinear fuzzy Fredholm integral equations using iterative method. Appl. Math. Comput. 225, 33-42 (2013)

22. Mirzaee, F: Numerical solution of Fredholm fuzzy integral equations of the second kind using hybrid of block-pulse-functions and Taylor series. Ain Shams Eng. J. 5, 631-636 (2014)

23. Dubois, D, Prade, H: Fuzzy numbers: an overview. In: Analysis of Fuzzy Information, vol. 1, pp. 3-39. CRC Press, Boca Raton (1987)

24. Wu, C, Gong, Z: On Henstock integral of fuzzy-number-valued functions (I). Fuzzy Sets Syst. 120, 523-532 (2001)

25. Bede, B, Stefanini, L: Generalized differentiability of fuzzy-valued functions. Fuzzy Sets Syst. 230, 119-141 (2013)

26. Bica, AM: Algebraic structures for fuzzy numbers from categorial point of view. Soft Comput. 11, 1099-1105 (2007)

27. Gal, SG: Approximation theory in fuzzy setting. In: Anastassiou, GA (ed.) Handbook of Analytic-Computational Methods in Applied Mathematics, pp. 617-666. Chapman \& Hall/CRC Press, Boca Raton (2000)

28. Stefanini, L: A generalization of Hukuhara difference and division for integral and fuzzy arithmetic. Fuzzy Sets Syst. $161,1564-1584(2010)$

29. Congxin, W, Cong, W: The supremum and infimum of the set of fuzzy numbers and its applications. J. Math. Anal. Appl. 210, 499-511 (1997)

30. Fang, J-X, Xue, Q-Y: Some properties of the space fuzzy-valued continuous functions on a compact set. Fuzzy Sets Syst. 160, 1620-1631 (2009)

31. Chalco-Cano, Y, Roman-Flores, $\mathrm{H}$ : On new solutions of fuzzy differential equations. Chaos Solitons Fractals 38 112-119 (2008)

32. Goetschel, R, Voxman, W: Elementary fuzzy calculus. Fuzzy Sets Syst. 18, 31-43 (1986)

33. Gong, Z, Wu, C: Bounded variation absolute continuity and absolute integrability for fuzzy-number-valued functions. Fuzzy Sets Syst. 129, 83-94 (2002)

34. Anastassiou, GA: Fuzzy Mathematics: Approximation Theory. Springer, Berlin (2010)

35. Jung, ZH, Schanfelberger, W: Block-Pulse Functions and Their Applications in Control Systems. Springer, Berlin (1992)

36. Maleknejad, K, Mahmoudi, Y: Numerical solution of linear Fredholm integral equation by using hybrid Taylor and Block-pulse functions. Appl. Math. Comput. 149, 799-806 (2004)

37. Mirzaee, F, Hoseini, AA: Numerical solution of nonlinear Volterra-Fredholm integral equations using hybrid of block-pulse functions and Taylor series. Alex. Eng. J. 52, 551-555 (2013) 